\title{
The ovulation trigger-OPU time interval of different ovarian protocols in ART: a retrospective study
}

\author{
Xi Shen ${ }^{1} \cdot$ Hui Long ${ }^{1} \cdot$ Wenya Guo $^{1} \cdot$ Yating Xie ${ }^{1} \cdot$ Hongyuan Gao $^{1} \cdot$ Jie Zhang $^{1} \cdot$ Yun Wang $^{1} \cdot$ Qifeng Lyu $^{1}$. \\ Yanping Kuang ${ }^{1} \cdot$ Li Wang $^{1}$ (D)
}

Received: 12 November 2019 / Accepted: 25 April 2020 / Published online: 3 June 2020

(c) The Author(s) 2020

\begin{abstract}
Purpose To explore the trends of oocyte and pregnancy outcomes over the ovulation trigger-OPU (oocyte pickup) time interval in four mainly used $\mathrm{COH}$ protocols.

Methods This retrospective study was conducted between January 2013 and July 2018. The IVF/ICSI cycles of the patients with normal ovarian reserve were included. The number of total patients was 4673, which consisted of long agonist protocol $(n=819)$, short agonist protocol $(n=1703)$, mild stimulation protocol $(n=1627)$, and GnRH antagonist protocol $(n=524)$. The primary outcome was mature oocyte rate.

Results The ovulation trigger-OPU time interval and $\mathrm{COH}$ protocol were related to cycles with $>80 \%$ MII oocytes. Four protocols showed apparently different trends of retrieved oocyte rate and mature oocyte rate over the ovulation trigger-OPU time interval, and the long agonist protocol had the most delayed time interval than other three $\mathrm{COH}$ protocols in retrieving more than $60 \%$ oocytes (35.4-39.6 h vs. 34.6-38.6 h vs. 32.5-37.5 h vs. 33.8-37.7 h) and getting more than $80 \%$ mature oocytes ( $35.0-39.7 \mathrm{~h}$ vs. $36.0-37.7 \mathrm{~h}$ vs. $34.1-35.5 \mathrm{~h}$ vs. $34.5-36.3 \mathrm{~h})$. And the adjusted odds ratio (OR) of the cumulative live birth rate (CLBR) (OR 1.360, 95\% Confidence Interval (CI) 1.156-1.549, $P<0.05$ ) significantly increased with the trigger-OPU time interval in the long agonist protocol.

Conclusions For getting more and mature oocytes, the ovulation trigger-OPU time intervals should be gradually prolonged from the mild stimulation protocol, the GnRH antagonist protocol, and the short protocol to the long agonist protocol. And the prolonged ovulation trigger-OPU time interval in the long agonist protocol brings higher live birth rate (LBR) and CLBR.
\end{abstract}

Keywords Ovulation trigger-OPU interval time $\cdot$ Retrieved oocyte rate $\cdot$ Mature oocyte rate $\cdot$ Controlled ovarian hyperstimulation protocol

Electronic supplementary material The online version of this article (https://doi.org/10.1007/s00404-020-05568-5) contains supplementary material, which is available to authorized users.

Yanping Kuang

kuangyanp@126.com

Li Wang

wanglishfd@126.com

1 Department of Assisted Reproduction, Shanghai Ninth People's Hospital Affiliated to Shanghai Jiao Tong University School of Medicine, Shanghai 200011, People's Republic of China

\section{Introduction}

The time interval from trigger to oocyte pickup (OPU) is vital, which consists of the luteinization start, the cumulus cell expansion, and the reduction division of the oocyte resumption [1]. In most in vitro fertilization (IVF) cycles, the commonly practiced interval was $32-36 \mathrm{~h}$, which was derived from the studies on patients who used Clomiphene Citrate (CC) and/or human menopausal gonadotropin (hMG) for ovulation induction $[2,3]$.

The clinical results of ART vary along with the time interval between oocyte maturation trigger and aspiration. Some studies have found that longer OPU time did not lead to more mature oocytes or better clinical results [4-8], whereas other studies have found that longer OPU time may produce more mature oocytes $[1,9,10]$, higher fertilization 
rate [11], better blastocyst development [12], more goodquality embryos, and higher ongoing pregnant rate [13]. There were also some studies which showed prolonging the interval between human chorionic gonadotropin (hCG) priming and oocyte retrieval could only increase the proportion of MII oocytes, rather than the pregnancy rates [14, 15]. Therefore, most research focused on whether doctors should delay the OPU time in the specific controlled ovarian hyperstimulation $(\mathrm{COH})$ protocol, such as the gonadotropinreleasing hormone analog (GnRHa) protocol.

A few studies researched the individual optimal ovulation trigger-OPU interval in the different $\mathrm{COH}$ protocols, which probably exist and need to be paid attention to, especially when $\mathrm{COH}$ protocols getting diverse. For example, GnRHa combined with hMG is different from the CC or hMG stimulation protocols; the former one needs longer oocyte OPU hours, usually 34-38 h [5, 9]. With current knowledge, there was only one meeting abstract firstly reporting the individual optimal trigger-OPU intervals of different $\mathrm{COH}$ protocols: $35-36 \mathrm{~h}$ in long protocol, 35-37 $\mathrm{h}$ in flare-up protocol, and $36-37 \mathrm{~h}$ in antagonist protocol [16].

Therefore, this retrospective analysis with a huge number of patients who used different $\mathrm{COH}$ protocols first explored the trends of oocyte and pregnancy outcomes over the ovulation trigger-OPU time interval in four mainly used $\mathrm{COH}$ protocols, to give a reference in arranging the ovulation trigger-OPU time intervals within patients using different $\mathrm{COH}$ protocols on the same day.

\section{Materials and methods}

\section{Study setting and patients}

This retrospective study was conducted between January 2013 and July 2018 in the Department of Assisted Reproduction of the Ninth People's Hospital affiliated to Shanghai Jiao Tong University School of Medicine. The following inclusion criteria were applied: women aged less than 40 years; basal follicle stimulating Hormone $(\mathrm{FSH})<10 \mathrm{~m} \mathrm{IU} / \mathrm{ml}$; antral follicle count $(\mathrm{AFC})>5$. The infertility reasons were tube factors, male factors, combined factors, and other factors (repeated intrauterine insemination failures and unknown reasons). Study following exclusion criteria was applied: (1) patients with endometriosis and polycystic ovarian syndrome; (2) $\mathrm{E}_{2}$ level on the day after trigger decrease more than $10 \%$ than the trigger day, which could induce the higher possibility of spontaneous premature ovulation and lower oocyte retrieval rate [17], the detailed number was as follows: 36 patients $(4.21 \%)$ in long agonist protocol; 49 patients $(2.80 \%)$ in short agonist protocol; 55 patients (3.27\%) in mild stimulation protocol; 23 patients (4.20\%) in GnRH antagonist protocol; (3) receipt of hormone treatments within the previous 3-month period and (4) any contraindications to ovarian stimulation treatment.

\section{Ovarian stimulation protocols}

The four $\mathrm{COH}$ regimens are described briefly as follows. In the long agonist protocol, the long-acting gonadotrophin-releasing agonist (leuprorelin acetate, $3.75 \mathrm{mg}$, Lizhu Pharmaceutical Trading Co., China) was administered on cycle days $2-5$. If downregulation was quantified $\left(\mathrm{E}_{2}<50 \mathrm{pg} / \mathrm{ml}\right)$ after 35 days, then hMG (150-225 IU/ day) was administered until trigger day. In short agonist protocol, patients were administered $0.1 \mathrm{mg}$ of short-acting gonadotrophin-releasing agonist (triptorelin, $0.1 \mathrm{mg}$, Ferring Pharmaceuticals, China) daily beginning on MC2 and 150-225 IU of hMG daily beginning on cycle day 3 . The mild ovarian stimulation protocols were flexible and consisted of letrozole $(2.5 \mathrm{mg}$, Jiangsu Hengrui Medicine Co. Ltd, Lianyungang, China), clomiphene $(50 \mathrm{mg} /$ day; Codal Synto Limited, Limassol, France) and hMG (75-150 IU) [18]. In GnRH-antagonist protocol, hMG (150-225 IU/day) was administered and the GnRH antagonist, cetrorelix (Pierre Fabre Medicament ProductionAquitaine Pharm International, France), was next administered daily by s.c. injection $(0.125-0.25 \mathrm{mg} /$ day $)$ when the diameter of one follicle reach $14 \mathrm{~mm}$ [19]. The doses were adjusted according to the transvaginal ultrasound examination. For all protocols, when three dominant follicles reached at least $18 \mathrm{~mm}$ or one dominant follicle reached at least $20 \mathrm{~mm}$ in diameter, the final oocyte maturation was triggered using Triptorelin $(0.1 \mathrm{mg}$, Ferring Pharmaceuticals) and/or hCG (5000 IU; Lizhu Pharmaceutical Trading Co., China) in mild stimulation protocol as well as $\mathrm{GnRH}$ antagonist protocol, while triggered by hCG (5000 IU) in short agonist protocol and long agonist protocol.

\section{Oocyte retrieval operation, insemination, and embryo culture}

Oocyte aspirations were conducted by one skillful physician in our center. All follicles more than $10 \mathrm{~mm}$ would be aspirated on the retrieved day. Aspirated oocytes were transferred to the embryology laboratory in Modified HTF Medium (Irvine Scientific, USA), and then, they were transferred to the culture medium. Fertilization was carried out via either IVF or intracytoplasmic sperm injection (ICSI), depending on the semen parameters of the patient's husband [20]. For IVF, fertilization was performed after about 4-6 $\mathrm{h}$ and the maturity was examined on the next day of OPU. In the situation of ICSI, oocytes were denuded and examined for maturity after $2-3 \mathrm{~h}$ preincubation. The immature oocytes were examined again 
after $2 \mathrm{~h}$ and all the mature oocytes were injected [21, 22]. According to the Cummins' standard, the third-day good-quality embryos were defined as grade 1 and grade 2 embryos with at least eight cells [23, 24]. Non-top-quality embryos were placed in extended culture until they reached the blastocyst stage. The good morphology blastocysts and the cleavage good-quality embryos were defined as viable embryos, which could be used for fresh or frozen embryo transfer (FET).

\section{Statistical analysis}

The primary outcome was mature oocyte rate. The second outcomes were oocyte retrieval rate, live birth rate, and cumulative live birth rate. The time interval of oocyte aspiration was defined as the midpoint of the start time (patient transferred to the operating table) and the end time (patient removed from the operating table). The following definitions were used: oocyte retrieval rate $=$ the number of cumulus-oocyte complex (COC) retrieved/the number of follicles $\geq 10 \mathrm{~mm}$ present on the retrieval day; mature oocyte rate $=$ the number of mature oocyte/the number of oocytes retrieved. Live births were defined as at least 22 gestational weeks or at least $500 \mathrm{~g}$. Cumulative live birth rate (CLBR) was defined as the number of deliveries with at least one live birth resulting from one aspirated ART cycle [25].

Statistical analyses were carried out using SPSS software (version 22, SPSS Inc., Chicago, IL, USA). Variables were expressed as the means $\pm \mathrm{SD}$, which were tested with oneway ANOVA. Qualitative data are presented as percentages and were tested with the Chi-squared test or Fisher's exact test when appropriate. A logistic regression model was performed to quantify the related factors of cycles with more than $80 \%$ mature oocytes and the odds ratio of pregnancyrelated outcomes. The adjusted odds ratio was calculated using the significant confounding factors selected from age, infertility duration, BMI, fertilization methods, AFC, and hMG doses. Additionally, locally estimated scatterplot smoothing (Loess) was adjusted with the $\mathrm{R}$ software ( $\mathrm{R}$ for windows, 3.4.4 version) to relate the percentage of oocyte retrieval and oocyte maturity trend over the lag trigger-OPU time interval. The time interval for getting more than $60 \%$ oocyte retrieval rate and $80 \%$ mature oocyte rate was defined as the junction of lower confidence interval limit [26] and reference line. Statistical significance was defined as a comparison resulting in $P<0.05$.

\section{Results}

\section{Related factors of cycles with more than $\mathbf{8 0 \%}$ MII oocytes}

A logistic regression analysis was performed to analyze the related factors of cycles with more than $80 \%$ MII oocytes (Table 1). The data illustrated that the factors of cycles with $>80 \%$ MII oocytes were not significantly related to age, BMI, and infertility duration $(P>0.05)$, nor with the oocyte trigger method [GnRH agonist as reference (ref.); hCG group: odds ratio (OR) 1.234, 95\% confidence interval (CI) 0.803-1.896, $P>0.05$; dual trigger group: OR 1.082, 95\% CI 0.719-1.629, $P>0.05]$. However, the antral follicle number (OR 0.981, 95\% CI 0.966-0.996, $P=0.017$ ) was a negative correlation factor and gonadotropin days (OR $1.038,95 \%$ CI $1.010-1.066 P=0.007$ ) were a positive factor of cycles more than $80 \%$ mature oocytes.
Table 1 Logistic regression analysis of factors associated with cycles with $>80 \%$ mature oocytes

\begin{tabular}{lllr}
\hline Parameter & & OR $(95 \% \mathrm{Cl})$ & $P$ value \\
\hline Age (years) & & $1.009(0.990-1.028)$ & 0.367 \\
Antral follicle count (n) & & $0.981(0.966-0.996)$ & 0.017 \\
Infertility duration (year) & & $0.984(0.953-1.016)$ & 0.312 \\
Body mass index (kg/m $\left.{ }^{2}\right)$ & & $0.977(0.951-1.004)$ & 0.092 \\
Gonadotropin days (day) & & $1.038(1.010-1.066)$ & 0.007 \\
Trigger-OPU time interval (h) & $<35.00$ & Reference & \\
& $35.01-36.00$ & $1.124(0.908-1.393)$ & 0.283 \\
& $36.01-37.00$ & $1.309(1.028-1.666)$ & 0.029 \\
& $37.01-38.00$ & $1.130(0.863-1.479)$ & 0.375 \\
& $>38.01$ & $1.265(0.908-1.761)$ & 0.165 \\
Ovulation stimulation protocol & Long agonist protocol & Reference & \\
& Short agonist protocol & $0.612(0.479-0.782)$ & $<0.001$ \\
& Mild stimulation protocol & $1.002(0.605-1.659)$ & 0.995 \\
& GnRH antagonist protocol & $0.815(0.542-1.225)$ & 0.326 \\
& GnRH agonist & Reference & \\
Ovulation trigger method & hCG & $1.234(0.803-1.896)$ & 0.338 \\
& Dual trigger (GnRH agonist + hCG) & $1.082(0.719-1.629)$ & 0.705 \\
\hline
\end{tabular}


Notably, there were another two significant factors related to cycles with $>80 \%$ MII oocytes. One was the oocyte aspiration interval time after triggering $(<35 \mathrm{~h}$ group as ref.; 35.01-36.00 h group: OR 1.124, 95\% CI $0.908-1.393, P>0.05 ; 36.01-37.00$ h group: OR 1.309 , 95\% CI 1.028-1.666, $P=0.029 ; 37.01-38.00$ group: OR $1.130,95 \%$ CI $0.863-1.479, P>0.05 ;>38.01$ h group: OR $1.265,95 \%$ CI $0.908-1.761, P>0.05)$. The other factor was the different $\mathrm{COH}$ protocols (long agonist protocol group as ref.; Short agonist protocol group: OR 0.612, 95\% Cl 0.479-0.782, $P<0.001$; Mild stimulation protocol group: OR 1.002, 95\% $\mathrm{Cl} 0.605-1.659, P>0.05$; and $\mathrm{GnRH}$ antagonist protocol group: OR $0.815,95 \% \mathrm{Cl} 0.542-1.225$, $P>0.05)$. These results indicated that the percentage of oocyte maturity was related to ovulation trigger-OPU time interval and $\mathrm{COH}$ protocol, which supported us to continue to explore the individual ovulation trigger-OPU time interval of four $\mathrm{COH}$ protocols.

\section{Four $\mathrm{COH}$ protocols showed different trends of oocyte retrieval rate along with their ovulation trigger-OPU time intervals}

Figure 1 showed a scatterplot for oocyte retrieval rate on the $y$-axis vs. the lag time on the $x$-axis in IVF/ICSI within
A Long agonist protocol $(\mathrm{N}=819)$

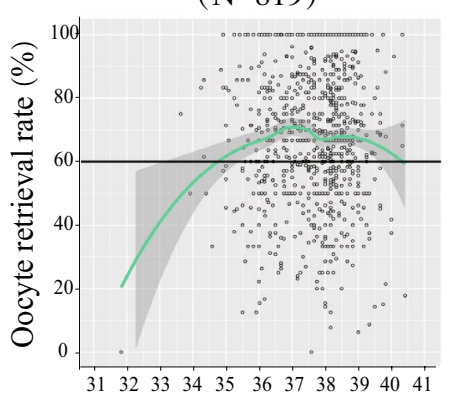

C Mild stimulation protocol $(\mathrm{N}=1627)$

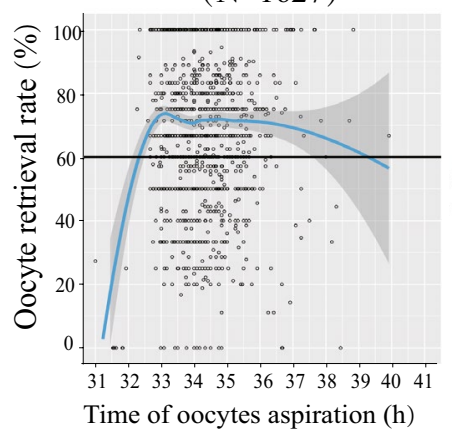

B Short agonist protocol

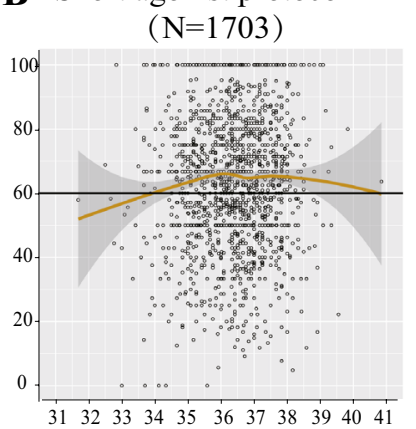

D GnRH antagonist protocol

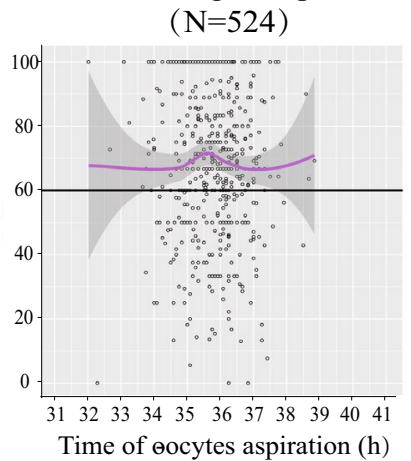

four $\mathrm{COH}$ protocols. Baseline characteristics of four $\mathrm{COH}$ protocols were first analyzed and comparable in the age, basal FSH, AFC, infertility duration, primary infertility, infertility reasons, BMI, and insemination methods (IVF, ICSI, IVF+ICSI) $(P>0.05)$ (Supplement Table 1).

However, the inherent characters of four $\mathrm{COH}$ protocols showed the apparent difference, such as gonadotropin $(\mathrm{Gn})$ doses, $\mathrm{E}_{2}$ level on the trigger day, and $\mathrm{E}_{2}$ level on the day after trigger day, which were also the key important regulators in ovulation. Therefore, four $\mathrm{COH}$ protocols had apparently different trends of oocyte retrieval rate along with their ovulation trigger-OPU time intervals in IVF/ ICSI (Fig. 1a-d) and ICSI (Sup Fig. 1 A1-D1). The oocyte retrieval rate was almost higher than $60 \%$ in the long agonist protocol (Fig. 1a, 35.4-39.6 h), short agonist protocol (Fig. 1b, 34.6-38.6 h), mild stimulation protocol (Fig. 1c, 32.5-37.5 h), and GnRH antagonist protocol (Fig. 1d, 33.8-37.7 h). The ovulation trigger-OPU time interval was most delayed in the long agonist protocol (Fig. 1e and Sup Fig. 1E1).

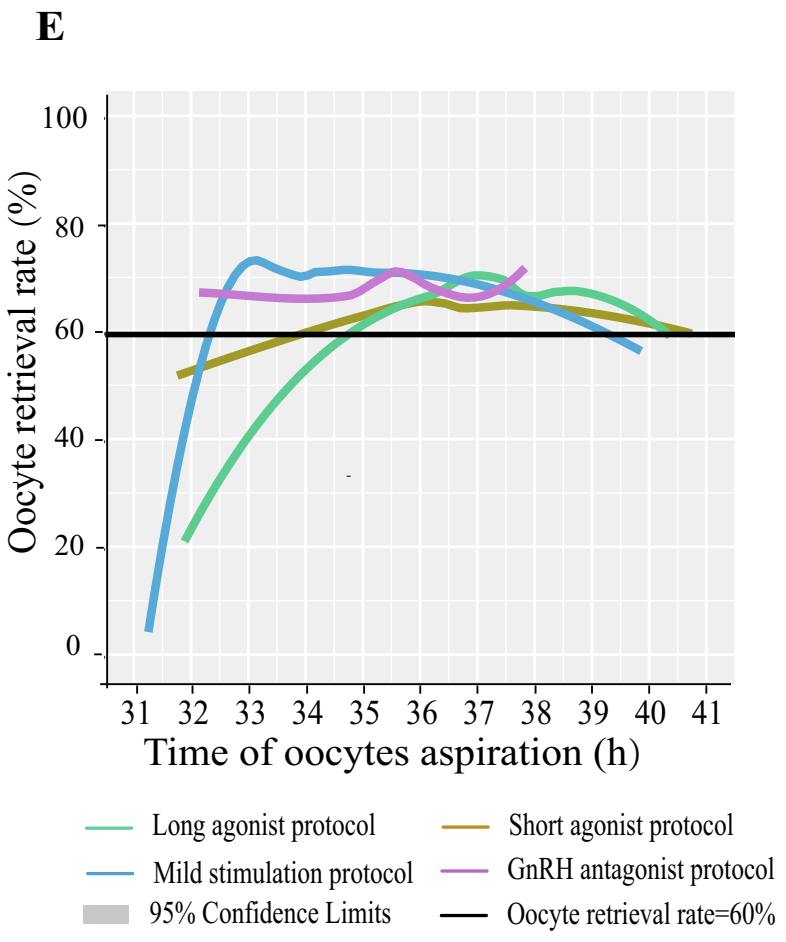

rately in the long agonist protocol, short agonist protocol, mild stimulation protocol, and GnRH antagonist protocol. e Merged image of a-d
Fig. 1 Scatterplot of four $\mathrm{COH}$ protocols demonstrating the oocyte retrieval as a function of lag time from ovulation trigger to ovum aspiration in IVF/ICSI, featuring loess trend with the $95 \%$ confidence interval highlighted with gray color. a-d Oocyte retrieval rate sepa- 


\section{The trends of mature oocyte rate were also different over the ovulation trigger-OPU time intervals of four $\mathrm{COH}$ protocols}

Except for the oocyte retrieval rate, four $\mathrm{COH}$ protocols also had different trends of mature oocyte rate over the lag time from ovulation trigger to ovum aspiration in IVF/ICSI (Fig. 2a-d) and ICSI (Sup Fig. 1 A2-D2). 80\% of mature oocyte rate was defined as a reference line, being not easy to achieve in the clinic. Seeing from the merged data (Fig. 2e and Sup Fig. 1 E2), the gradual delayed tendency of ovulation trigger-OPU time intervals from the mild stimulation protocol $(34.1-35.5 \mathrm{~h}$ in IVF/ ICSI; $32.8-35.3 \mathrm{~h}$ in ICSI), the GnRH antagonist protocol (34.5-36.3 $\mathrm{h}$ in IVF/ICSI; None in ICSI), and the short protocol (36.0-37.7 $\mathrm{h}$ in IVF/ICSI; $35.1-37.2 \mathrm{~h}$ in ICSI) to the long agonist protocol (35.0-39.7 $\mathrm{h}$ in IVF/ ICSI; $35.5-37.5 \mathrm{~h}$ in ICSI), was also existed in the mature oocyte rate.
A Long agonist protocol

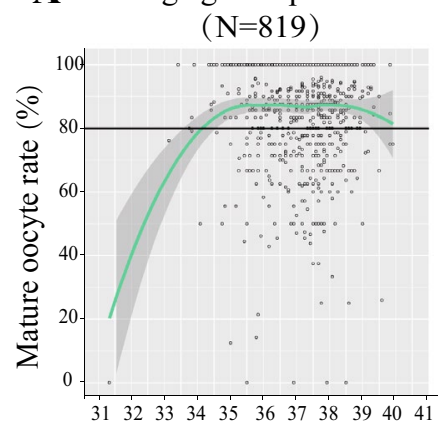

C Mild stimulation protocol $(\mathrm{N}=1627)$

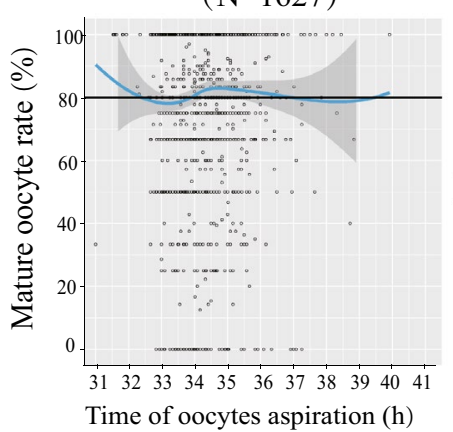

B Short agonist protocol

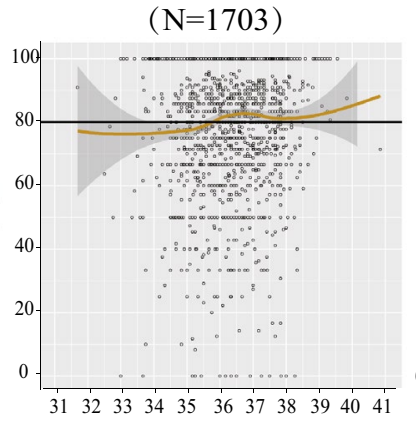

D GnRH antagonist protocol

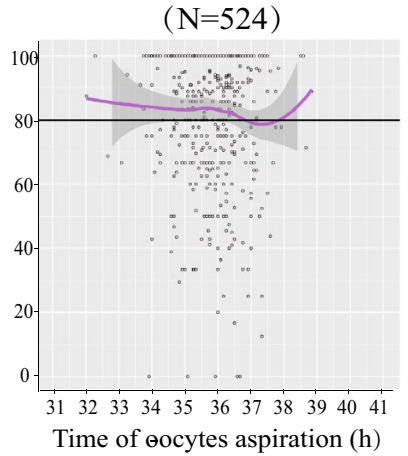

Fig. 2 Scatterplot of four $\mathrm{COH}$ protocols demonstrating the mature oocyte rate as a function of lag time from ovulation trigger to ovum aspiration in IVF/ICSI, featuring loess trend with the 95\% confidence interval highlighted with gray color. a-d Mature oocyte rate

\section{The summary of pregnancy outcomes over the ovulation trigger-OPU time intervals in four $\mathrm{COH}$ protocols}

Unlike the oocyte retrieval rate and mature oocyte rate, not all the pregnancy outcomes are closely related to the ovulation trigger -OPU time intervals in four $\mathrm{COH}$ protocols (Table 2). After adjusting confounders, comparing with the implantation rate and clinical pregnancy rate, only the adjusted live birth rate per transfer (OR $1.196,95 \%$ CI $1.045-1.368, P<0.05)$ and the CLBR (OR $1.360,95 \% \mathrm{CI}$ $1.156-1.549, P<0.05)$ had positive relations with their trigger-OPU time intervals of long agonist protocol in four $\mathrm{COH}$ protocols.

Table 3 shows the overview of ovulation trigger-OPU time intervals in four $\mathrm{COH}$ protocols. This timing advance tendency of mild stimulation protocol and GnRH antagonist protocol was seen from the $80 \%$ concentrated time intervals, which was further reflected in the ovulation trigger-OPU time period with more than $60 \%$ oocyte retrieval rate and more than $80 \%$ mature oocyte rate. And the long agonist protocol had the most delayed ovulation trigger-OPU time interval with $>60 \%$ oocyte retrieval rate (35.4-39.6 h, $4.2 \mathrm{~h}$ ) and $>80 \%$ mature oocyte rate (35.0-39.7 h, $4.7 \mathrm{~h})$.

$\mathbf{E}$

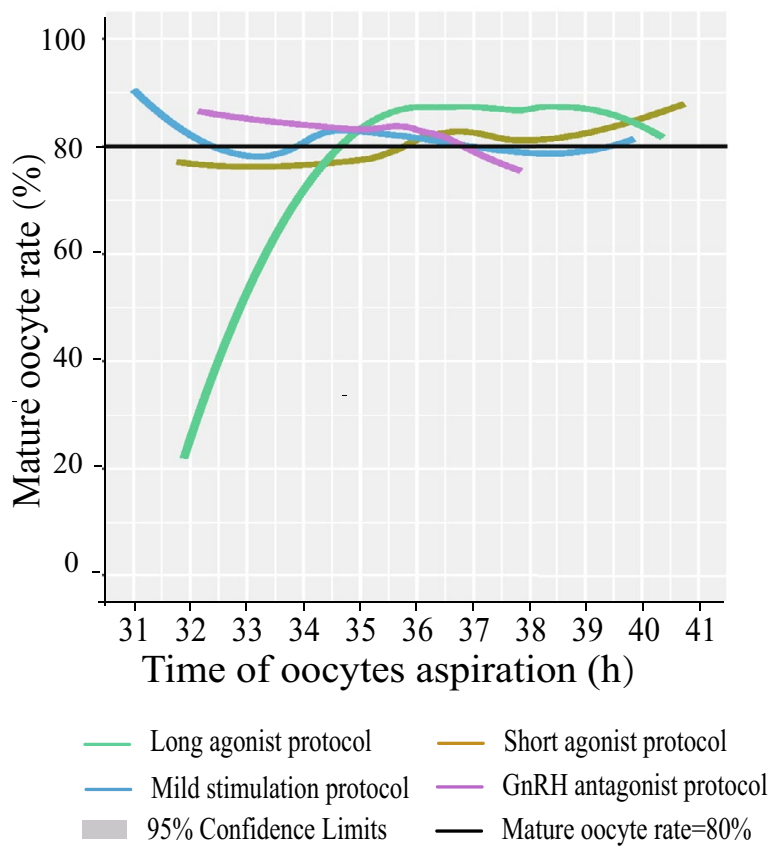

separately in the long agonist protocol, short agonist protocol, mild stimulation protocol, and GnRH antagonist protocol. e Merged image of $\mathbf{a}-\mathbf{d}$ 
Table 2 The odds ratio of pregnancy outcomes with trigger-OPU time interval

\begin{tabular}{lllll}
\hline & Crude OR (95\% CI) & $P$ & Adjusted OR (95\% CI) & $P$ \\
\hline Clinical pregnancy rate & & & & \\
Long agonist protocol & $1.081(0.956-1.223)$ & 0.213 & $1.129(0.976-1.305)$ & 0.102 \\
Short agonist protocol & $0.955(0.876-1.041)$ & 0.297 & $0.971(0.883-1.067)$ & 0.535 \\
Mild stimulation protocol & $1.085(0.963-1.223)$ & 0.181 & $1.096(0.967-1.242)$ & 0.150 \\
GnRH antagonist protocol & $0.806(0.656-0.990)$ & 0.040 & $0.860(0.685-1.063)$ & 0.163 \\
Implantation rate & & & & \\
Long agonist protocol & $1.045(0.943-1.157)$ & 0.405 & $1.083(0.960-1.221)$ & 0.194 \\
Short agonist protocol & $0.964(0.897-1.035)$ & 0.311 & $0.951(0.885-1.021)$ & 0.167 \\
Mild stimulation protocol & $1.065(0.966-1.175)$ & 0.205 & $0.850(0.707-1.021)$ & 0.082 \\
GnRH antagonist protocol & $0.816(0.689-0.968)$ & 0.019 & $0.867(0.728-1.033)$ & 0.111 \\
Live birth rate per transfer & & & & \\
Long agonist protocol & $1.181(1.033-1.350)$ & 0.015 & $1.196(1.045-1.368)$ & 0.009 \\
Short agonist protocol & $0.977(0.892-1.071)$ & 0.622 & $1.013(0.988-1.038)$ & 0.318 \\
Mild stimulation protocol & $1.091(0.966-1.232)$ & 0.162 & $1.034(0.901-1.187)$ & 0.631 \\
GnRH antagonist protocol & $0.878(0.709-1.086)$ & 0.230 & $0.940(0.754-1.171)$ & 0.580 \\
Cumulative live birth rate & & & & 0.020 \\
Long agonist protocol & $1.311(1.143-1.502)$ & 0.000 & $1.360(1.156-1.549)$ & 0.471 \\
Short agonist protocol & $1.033(0.942-1.132)$ & 0.495 & $0.947(0.816-1.098)$ & 0.200 \\
Mild stimulation protocol & $1.215(1.086-1.359)$ & 0.001 & $1.088(0.956-1.237)$ & 0.163 \\
GnRH antagonist protocol & $0.794(0.645-0.978)$ & 0.030 & $0.859(0.695-1.063)$ & \\
\hline
\end{tabular}

Table 3 Description of trigger-OPU time period in different $\mathrm{COH}$ protocols

\begin{tabular}{|c|c|c|c|c|c|}
\hline & $\begin{array}{l}\text { Total trigger- } \\
\text { OPU time period } \\
\text { (h) }\end{array}$ & $\begin{array}{l}\text { Trigger-OPU time period } \\
\text { (concentrated in 10th- } \\
\text { 90th, h) }\end{array}$ & $\begin{array}{l}\text { Trigger-OPU time period } \\
(>60 \% \text { oocyte retrieval } \\
\text { rate, } \mathrm{h})\end{array}$ & $\begin{array}{l}\text { Trigger-OPU time period } \\
(>80 \% \text { mature oocyte } \\
\text { rate, } \mathrm{h})\end{array}$ & $\begin{array}{l}\text { Relationship } \\
\text { with LBR and } \\
\text { CLBR }\end{array}$ \\
\hline Long agonist protocol & $31.8-40.4(8.6)$ & $36.0-39.0(3.0)$ & $35.4-39.6(4.2)$ & $35.0-39.7(4.7)$ & Positive \\
\hline Short agonist protocol & $31.7-40.9(9.2)$ & $35.0-38.0(3.0)$ & $34.6-38.6(4.0)$ & $36.0-37.7(1.7)$ & None \\
\hline Mild stimulation protocol & $30.4-39.9(9.5)$ & $33.0-35.5(2.5)$ & $32.5-37.5(5.0)$ & $34.1-35.5(1.4)$ & None \\
\hline $\begin{array}{l}\text { GnRH antagonist pro- } \\
\text { tocol }\end{array}$ & $32.0-38.8(6.8)$ & $34.5-37.0(2.5)$ & $33.8-37.7(3.9)$ & $34.5-36.3(1.8)$ & None \\
\hline
\end{tabular}

\section{Discussion}

This study first explored that the ovulation trigger-OPU time interval and $\mathrm{COH}$ protocol are two main factors relating to cycles more than $80 \%$ MII oocytes. To retrieve more than $60 \%$ oocytes and get more than $80 \%$ mature oocytes, the ovulation trigger-OPU time intervals of four $\mathrm{COH}$ cycles were apparently different and had a gradual delayed tendency among the mild stimulation protocol, GnRH antagonist protocol, short agonist protocol, and the long agonist protocol. Moreover, the ovulation trigger-OPU time interval was positively corelated with the cumulative live birth rate in the long agonist protocol, rather than other three $\mathrm{COH}$ protocols.

\section{Strengths and limitations}

This research first overviews that four $\mathrm{COH}$ protocols which are usually used in the clinic at present have their individual explicit features of ovulation trigger-OPU time intervals, not only considering from the oocytes performance such as oocyte retrieval rate and mature oocyte rate, also from pregnancy outcomes such as LBR and CLBR. Actually, there is a lack of specialized and systematic research on this aspect. Furthermore, former studies usually divided their trigger-OPU time into several subgroups and focused on the diversity between the shorter period and longer period [5, 9]. However, our study pays attention to the effect of continuous time interval on clinical 
outcomes, which could take advantage of more useful and intuitive information.

Our study also has some limitations. This is a retrospective study without randomization. Second, we analyzed the relationship of ovulation trigger-OPU time interval with the pregnancy outcomes in four $\mathrm{COH}$ protocols. However, the transfer strategy of four $\mathrm{COH}$ protocols are different: the GnRH antagonist and long protocols are matched with both fresh embryo transfer (ET) and FET, while the short protocol and the mild stimulation protocol used FET, and sometimes, ET would be chosen. Therefore, we calculated not only the live birth rate, but also the cumulative live birth rate (CLBR), which included the ET and subsequent FET cycles. However, the randomized-controlled research combined with the same transfer method is need to explore this question in the future.

\section{Comparisons of results between ours and previous studies}

Some studies considered that mature oocyte rate had no significant relationship with basic parameters [14]. In our research, we found that there were some basic parameters associating with mature oocyte rate. Interestingly, AFC is negatively relative with mature oocyte rate. The possible reason may be that although higher AFC could lead to more retrieved oocytes, they also contributed to higher proportion of small immature oocytes on the retrieval day, which may result in lower oocyte maturity rate. Because Anti-Mullerian Hormone (AMH), which is another indicator of ovarian reserves and strong positively with AFC, also had higher correlation coefficients with immature oocytes than mature oocytes [27, 28].

Previous research mostly explored the optimal ovulation trigger-OPU time interval in one specific $\mathrm{COH}$ protocol. The early research found the proper trigger-OPU interval in patients stimulated with clomiphene citrate (CC) or hMG was 32-36 $\mathrm{h}[2,29,30]$. After that, lots of research explored whether oocytes should be retrieved earlier or later in the gonadotropin-releasing hormone analog $(\mathrm{GnRHa})$ combined with hMG protocol $[1,5,9,13,14,31,32]$. For the different GnRHa protocols which may bring the distinct extent of pituitary suppression and need diverse in vivo maturation time before oocyte retrieving, the only related research is one meeting abstract [16], which found the long protocol, flare-up, and antagonist protocols had their discrepant optimal time for oocyte retrieval in terms of egg maturation, fertilization, implantation, and clinical pregnancy rates. Our research further illustrated the diversity of trends in oocyte and pregnancy outcomes with trigger-OPU time interval in four $\mathrm{COH}$ protocols mainly used at present.

We explored the trends of oocyte retrieval rate and mature oocyte rate along with the ovulation trigger-OPU time intervals mainly in IVF/ISCI, but also in ICSI. The oocyte maturity in ICSI is an important and necessary supplement to evaluate the features of ovulation trigger-OPU time intervals in those $\mathrm{COH}$ cycles. The ovulation trigger-OPU time periods were also apparently different in the four $\mathrm{COH}$ protocols and showed the delaying tendency from the mild stimulation protocol, GnRH antagonist protocol to the short and long agonist protocol, same as in the IVF/ICSI. Some groups had limited sample sizes such as GnRH antagonist protocol with ICSI ( $N=157$, Supplement Fig. 1 D2 and E2), which may lead to some biases and the worst outcome in long time interval. A more accurate evaluation of oocyte performance would be performed with larger sample size.

\section{Possible mechanisms of the individual optimal ovulation trigger-OPU time intervals of different $\mathrm{COH}$ protocols}

As the classic protocols used all over the world, the GnRH analogs had lots of types. The short agonist protocol, long agonist protocol, and $\mathrm{GnRH}$ antagonist protocol all suppress $\mathrm{E}_{2}$-positive feedback and the preovulatory $\mathrm{GnRH} / \mathrm{LH}$ surge in the pituitary, but the suppression time period is different. The GnRH antagonist is administrated after the oocytes growing up, while the long agonists are usually administrated 1 month before ovarian hyperstimulation and short agonists are initially injected at the same time with $\mathrm{hMG}$ [33], which may bring the different extent of pituitary suppression. Therefore, the ovulation trigger-OPU time intervals for getting over $80 \%$ mature oocytes seem having the gradual prolonged tendency among the GnRH antagonist protocol (34.5-36.3 h), the short protocol (36.0-37.7 h), and the long agonist protocol (35.0-39.7 h).

Consistent with our clinical experience, the long agonist protocol has the most delayed ovulation trigger-OPU time interval. And the explanation may be that the lowest $\mathrm{LH}$ level on trigger day in long agonist protocol (Supplement Table 1). When the same doses of hCG act on LH receptors, the lowest LH level may need more time to finish the process of cumulus cell oocyte complex (COC) expansion. Moreover, the lower follicular LH level may result in higher sensitivity of LH receptors, which leads to higher concentration of amphiregulin and promotes oocyte maturation [34].

Furthermore, the mild stimulation protocol had a relatively earlier trend in retrieving oocytes, owing to its specific inherent characters in superovulation. The usage of CC has the antiestrogenic effect, which may suppress premature LH surge. However, this inhibition function of CC is less effective than GnRH analog, which could also be seen in the higher cancelation rate [35], so we usually pick up the oocytes early to avoid the preovulation. Our study also indicates that the highest oocyte retrieval rate was observed between 32 and $33 \mathrm{~h}$; however, the mature oocyte rate was 
obviously higher in later time period (Figs. 1c, 2c). This may explain why we usually retrieve the oocytes early enough to avoid preovulation in mild stimulation protocol, but which may impair the cytoplasmic maturation and oocyte development competence and bring this unsatisfactory oocyte maturation.

Last but not least, not only LBR per transfer but also CLBR is concerned due to FET cycles. According to Deng et al. [10], the extended time interval between hCG administration and oocyte retrieval might increase the cumulative live birth rate of embryos from a cohort of oocytes retrieved during one cycle when adopting FET strategy. Longer ovulation trigger-OPU time interval could bring out a higher trend in oocyte retrieval rate and mature oocyte rate, which would lead to more mature oocytes in later time period and more subsequent transplant FET cycles, thus resulting in higher CLBR. However, no significant increase was observed in the other three protocols. Our study shows that the trigger-OPU time interval related more closely with oocyte outcomes than pregnancy outcomes. This may be due to that the trigger-OPU interval could partly influence nuclear and cytoplasmic maturity of oocytes, but more factors would take part in the later process of embryonic development and implantation [36].

\section{Conclusion}

The ovulation trigger-OPU time interval and $\mathrm{COH}$ protocol are two main factors relating to cycles more than $80 \%$ mature oocytes. The ovulation trigger-OPU intervals for retrieving $>60 \%$ oocytes and getting $>80 \%$ maturity are apparently different in four $\mathrm{COH}$ protocols, and had a gradual prolonged tendency from the mild stimulation protocol, the GnRH antagonist protocol, and the short protocol to the long agonist protocol. Moreover, the prolonged ovulation trigger-OPU intervals had a significantly positive relation with a live birth rate per transfer and cumulative live birth rate only in the long agonist protocol.

Acknowledgements We gratefully acknowledge all the doctors, nurses, and laboratory staffs employed in the Department of Assisted Reproduction, Shanghai Ninth People's Hospital Affiliated to Shanghai Jiao Tong University School of Medicine.

Author contributions LW and YPK supervised the entire study, including the procedures, conception, design, and completion. XS: acquisition of data, analysis and interpretation of data, and drafting the article. HL, WYG, XYT, HYG, and JZ: acquisition of data and revising the article. YW and QFL: data analysis. All authors approved the final version of the manuscript.

Funding The study was financially supported by the National Natural Science Foundation of China (Grant number: 31400970 to L. W., 81771533 to Y. K.), the National Key project (Grant number:
2018YFC1003000 to Y. K.), and the grants from the Natural Science Foundation of Shanghai (Grant number: 18ZR1422600 to L. W.),

Conflict of interest None declared.

Ethics approval This study was approved by the Ethics Committee of Shanghai Ninth People's Hospital (Institutional Review Board).

Informed consent All patients have provided their consent for the data to be used for research.

Open Access This article is licensed under a Creative Commons Attribution 4.0 International License, which permits use, sharing, adaptation, distribution and reproduction in any medium or format, as long as you give appropriate credit to the original author(s) and the source, provide a link to the Creative Commons licence, and indicate if changes were made. The images or other third party material in this article are included in the article's Creative Commons licence, unless indicated otherwise in a credit line to the material. If material is not included in the article's Creative Commons licence and your intended use is not permitted by statutory regulation or exceeds the permitted use, you will need to obtain permission directly from the copyright holder. To view a copy of this licence, visit http://creativecommons.org/licenses/by/4.0/.

\section{References}

1. Gudmundsson J, Fleming R, Jamieson ME, McQueen D, Coutts JR (1990) Luteinization to oocyte retrieval delay in women in whom multiple follicular growth was induced as part of an in vitro fertilization/gamete intrafallopian transfer program. Fertil Steril 53(4):735-737. https://doi.org/10.1016/s0015-0282(16)53474-8

2. Edwards RG, Steptoe PC (1975) Induction of follicular growth, ovulation and luteinization in the human ovary. J Reprod Fertil Suppl 22:121-163

3. Testart J, Frydman R (1982) Minimum time lapse between luteinizing hormone surge or human chorionic gonadotropin administration and follicular rupture. Fertil Steril 37(1):50-53. https://doi. org/10.1016/s0015-0282(16)45976-5

4. Droesch K, Muasher SJ, Kreiner D, Jones GS, Acosta AA, Rosenwaks Z (1988) Timing of oocyte retrieval in cycles with a spontaneous luteinizing hormone surge in a large in vitro fertilization program. Fertil Steril 50(3):451-456

5. Nargund G, Reid F, Parsons J (2001) Human chorionic gonadotropin-to-oocyte collection interval in a superovulation IVF program. A prospective study. J Assist Reprod Genet 18(2):87-90. https:// doi.org/10.1023/a:1026530624575

6. Bjercke S, Tanbo T, Dale PO, Abyholm T (2000) Comparison between two HCG-to-oocyte aspiration intervals on the outcome of IVF. Hum Reprod 15(1):227-228. https://doi.org/10.1093/ humrep/15.1.227-a

7. Bosdou JK, Kolibianakis EM, Venetis CA, Zepiridis L, Chatzimeletiou K, Makedos A, Triantafyllidis S, Masouridou S, Mitsoli A, Tarlatzis B (2015) Is the time interval between HCG administration and oocyte retrieval associated with oocyte retrieval rate? Reprod Biomed Online 31(5):625-632. https://doi.org/10.1016/j. rbmo.2015.08.005

8. Garor R, Shufaro Y, Kotler N, Shefer D, Krasilnikov N, BenHaroush A, Pinkas H, Fisch B, Sapir O (2015) Prolonging oocyte in vitro culture and handling time does not compensate for a shorter interval from human chorionic gonadotropin administration to oocyte pickup. Fertil Steril 103(1):72-75. https://doi. org/10.1016/j.fertnstert.2014.09.022 
9. Mansour RT, Aboulghar MA, Serour GI (1994) Study of the optimum time for human chorionic gonadotropin-ovum pickup interval in in vitro fertilization. J Assist Reprod Genet 11(9):478-481. https://doi.org/10.1007/bf02215712

10. Deng M, Liang Y, Qin H, Tan Y, Mai Q, Yuan X, Yuan Y (2019) A moderately extended time interval between $\mathrm{hCG}$ administration and oocyte retrieval is good for most patients with oocyte retrieval scheduled on the same day: a retrospective cohort study. J Obstet Gynaecol. https://doi.org/10.1080/01443615.2019.1679735

11. Jamieson ME, Fleming R, Kader S, Ross KS, Yates RW, Coutts JR (1991) In vivo and in vitro maturation of human oocytes: effects on embryo development and polyspermic fertilization. Fertil Steril 56(1):93-97. https://doi.org/10.1016/s0015-0282(16)54424-0

12. Papayannis M, Demarco A, Terrado Gil G, Bisioli C, Serna J, Gomez Pena M, Rocha M, De Zuniga I, Kopcow L, Sobral F (2018) Time intervals from the hCG trigger: analysis of different checkpoints and their impact on embryo development, implantation and pregnancy. In: Abstracts of the 34th Annual Meeting of the European Society of Human Reproduction and Embryology, vol 33, Suppl 1, p i197. https://doi.org/10.1093/humrep/33.Supplement_1.1

13. Tadros T, Chenoz L, Poulain M, Adda-Herzog E, Ayoubi J, Fanchin R (2017) Time interval between hCG administration and oocyte retrieval significantly influences IVF-ET results. In: Abstracts of the 33rd Annual Meeting of the. European Society of Human Reproduction and Embryology, vol 32, Suppl 1, pp i77-i78. https://doi.org/10.1093/humrep/32.Supplement_1.1

14. Weiss A, Neril R, Geslevich J, Lavee M, Beck-Fruchter R, Golan J, Shalev E (2014) Lag time from ovulation trigger to oocyte aspiration and oocyte maturity in assisted reproductive technology cycles: a retrospective study. Fertil Steril 102(2):419-423. https ://doi.org/10.1016/j.fertnstert.2014.04.041

15. Wang W, Zhang XH, Wang WH, Liu YL, Zhao LH, Xue SL, Yang KH (2011) The time interval between hCG priming and oocyte retrieval in ART program: a meta-analysis. J Assist Reprod Genet 28(10):901-910. https://doi.org/10.1007/s10815-011-9613-x

16. Tien NV, Hoi NX (2014) Optimizing the time interval between hCG triggering and occyte retrieval in different ovarian protocols in art: a prospective study in Vietnam. Fertil Steril 102(3, Supplement):e315. https://doi.org/10.1016/j.fertnstert .2014.07.1069

17. Lu X, Khor S, Zhu Q, Sun L, Wang Y, Chen Q, Wu L, Fu Y, Tian H, Lyu Q, Cai R, Kuang Y (2018) Decrease in preovulatory serum estradiol is a valuable marker for predicting premature ovulation in natural/unstimulated in vitro fertilization cycle. J Ovarian Res 11(1):96. https://doi.org/10.1186/s13048-018-0469-X

18. Wang N, Wang Y, Chen Q, Dong J, Tian H, Fu Y, Ai A, Lyu Q, Kuang Y (2016) Luteal-phase ovarian stimulation vs conventional ovarian stimulation in patients with normal ovarian reserve treated for IVF: a large retrospective cohort study. Clin Endocrinol (Oxf) 84(5):720-728. https://doi.org/10.1111/cen.12983

19. Wang Y, Kuang Y, Chen Q, Cai R (2018) Gonadotropin-releasing hormone antagonist versus progestin for the prevention of premature luteinising hormone surges in poor responders undergoing in vitro fertilisation treatment: study protocol for a randomised controlled trial. Trials 19(1):455. https://doi.org/10.1186/s1306 3-018-2850-x

20. Henkel RR, Schill WB (2003) Sperm preparation for ART. Reprod Biol Endocrinol 1:108. https://doi.org/10.1186/1477-7827-1-108

21. Salha O, Nugent D, Dada T, Kaufmann S, Levett S, Jenner L, Lui S, Sharma V (1998) The relationship between follicular fluid aspirate volume and oocyte maturity in in-vitro fertilization cycles. Hum Reprod 13(7):1901-1906

22. Rienzi L, Vajta G, Ubaldi F (2011) Predictive value of oocyte morphology in human IVF: a systematic review of the literature. Hum Reprod Update 17(1):34-45. https://doi.org/10.1093/humup d/dmq029
23. Cummins JM, Breen TM, Harrison KL, Shaw JM, Wilson LM, Hennessey JF (1986) A formula for scoring human embryo growth rates in in vitro fertilization: its value in predicting pregnancy and in comparison with visual estimates of embryo quality. J In Vitro Fert Embryo Transf 3(5):284-295

24. Kuang Y, Chen Q, Fu Y, Wang Y, Hong Q, Lyu Q, Ai A, Shoham $Z$ (2015) Medroxyprogesterone acetate is an effective oral alternative for preventing premature luteinizing hormone surges in women undergoing controlled ovarian hyperstimulation for in vitro fertilization. Fertil Steril 104(1):62 e63-70 e63. https:// doi.org/10.1016/j.fertnstert.2015.03.022

25. Zegers-Hochschild F, Adamson GD, Dyer S, Racowsky C, de Mouzon J, Sokol R, Rienzi L, Sunde A, Schmidt L, Cooke ID, Simpson JL, van der Poel S (2017) The international glossary on infertility and fertility care. Hum Reprod 32(9):1786-1801. https ://doi.org/10.1093/humrep/dex234

26. Ozarda Y, Sikaris K, Streichert T, Macri J, Intervals ICoR, Decision L (2018) Distinguishing reference intervals and clinical decision limits-a review by the IFCC committee on reference intervals and decision limits. Crit Rev Clin Lab Sci 55(6):420-431. https://doi.org/10.1080/10408363.2018.1482256

27. Jee BC, Ku SY, Suh CS, Kim KC, Lee WD, Kim SH, Seoul National University College of Medicine Assisted Reproductive Technology Study G (2008) Serum anti-Mullerian hormone and inhibin B levels at ovulation triggering day can predict the number of immature oocytes retrieved in in vitro fertilization cycles. J Korean Med Sci 23(4):657-661. https://doi.org/10.3346/ jkms.2008.23.4.657

28. Sayme N, Kljajic M, Krebs T, Maas DHA (2019) The impact of anti-Mullerian hormone (AMH) on multiple pronuclei (PN) presence and oocyte maturity in ICSI treatments. Gynecol Endocrinol. https://doi.org/10.1080/09513590.2019.1706080

29. Mulnard J (1982) In vitro fertilization and embryo transfer as a treatment in tubal sterility. The first developmental stages of the human embryo: from fertilization to nidation. Rev Med Brux 3(4):275-284

30. Edwards RG, Steptoe PC (1974) Control of human ovulation, fertilization and implantation. Proc R Soc Med 67(9):932-936

31. Raziel A, Schachter M, Strassburger D, Kasterstein E, Ron-El $\mathrm{R}$, Friedler S (2006) In vivo maturation of oocytes by extending the interval between human chorionic gonadotropin administration and oocyte retrieval. Fertil Steril 86(3):583-587. https://doi. org/10.1016/j.fertnstert.2006.02.091

32. Bjercke S, Tanbo T, Dale PO, Abyholm T (2000) Comparison between two hCG-to-oocyte aspiration intervals on the outcome of in vitro fertilization. J Assist Reprod Genet 17(6):319-322. https ://doi.org/10.1023/a:1009401027251

33. van Loenen AC, Huirne JA, Schats R, Hompes PG, Lambalk CB (2002) GnRH agonists, antagonists, and assisted conception. Semin Reprod Med 20(4):349-364. https://doi. org/10.1055/s-2002-36713

34. Nana L, Yanmin M, Rong L, Hongyan J, Min L, Xiang H, Feng HL, Jie Q (2012) Comparison of follicular fluid amphiregulin and EGF concentrations in patients undergoing IVF with different stimulation protocols. Endocrine 42(3):708-716

35. Alper MM, Fauser BC (2017) Ovarian stimulation protocols for IVF: is more better than less? Reprod Biomed Online 34(4):345353. https://doi.org/10.1016/j.rbmo.2017.01.010

36. Ghasemian F, Faraji R, Asgharnia M, Zahiri Z, Bahadori MH (2013) The impact of different time intervals between hCG priming and oocyte retrieval on ART outcomes. Iran J Reprod Med 11(7):559-564

Publisher's Note Springer Nature remains neutral with regard to jurisdictional claims in published maps and institutional affiliations. 